Mircea Sofonea (Perpignan)

Mohamed Ait Mansour (Limoges)

\title{
A CONVERGENCE RESULT FOR EVOLUTIONARY VARIATIONAL INEQUALITIES AND APPLICATIONS TO ANTIPLANE FRICTIONAL CONTACT PROBLEMS
}

Abstract. We consider a class of evolutionary variational inequalities depending on a parameter, the so-called viscosity. We recall existence and uniqueness results, both in the viscous and inviscid case. Then we prove that the solution of the inequality involving viscosity converges to the solution of the corresponding inviscid problem as the viscosity converges to zero. Finally, we apply these abstract results in the study of two antiplane quasistatic frictional contact problems with viscoelastic and elastic materials, respectively. For each of the problems we prove the existence of a unique weak solution; we also provide convergence results, together with their mechanical interpretation.

1. Introduction. Let $V$ be a real Hilbert space endowed with the inner product $(\cdot, \cdot)_{V}$ and the associated norm $\|\cdot\|_{V}$, and let $T>0$. Consider two operators $A: V \rightarrow V$ and $B: V \rightarrow V$, a functional $j: V \rightarrow \mathbb{R}$ and a function $f:[0, T] \rightarrow V$. Everywhere in this paper a dot above a variable will represent the derivative with respect to time. Let $\theta$ be a positive parameter, called viscosity, and let $u_{0} \in V$. With these data we consider the following problem:

Problem $P_{\theta}$. Find a function $u_{\theta}:[0, T] \rightarrow V$ such that

$$
\begin{aligned}
\theta\left(A \dot{u}_{\theta}(t), v-\dot{u}_{\theta}(t)\right)_{V}+\left(B u_{\theta}(t), v-\dot{u}_{\theta}(t)\right)_{V}+j(v)-j\left(\dot{u}_{\theta}(t)\right) \\
\geq \\
\geq\left(f(t), v-\dot{u}_{\theta}(t)\right)_{V} \quad \forall v \in V, \text { a.e. } t \in(0, T), \\
u(0)=u_{0} .
\end{aligned}
$$

2000 Mathematics Subject Classification: 74M10, 74M15, 49J40.

Key words and phrases: evolutionary inequality, viscosity coefficient, antiplane problem, viscoelastic material, elastic material, frictional contact, weak solution. 
Problems of this form arise in the study of variational models for frictional contact with Kelvin-Voigt viscoelastic materials (see for instance $[3,4])$. There, $u$ represents the displacement field, $A$ and $B$ are the viscosity and elasticity operators, respectively, and the functional $j$ is determined by the type of contact and friction boundary conditions. The function $f$ is related to the given body forces and surface tractions, and $u_{0}$ represents the initial displacement. Notice that usually the functional $j$ in (1.1) is nondifferentiable.

Two questions arise in the study of Problem $P_{\theta}$. The first one concerns the unique solvability of this problem and it was discussed in [3]. There, a general existence and uniqueness result for a class of variational inequalities which contains problem (1.1)-(1.2) as a special case was provided. Assume now that Problem $P_{\theta}$ has a unique solution $u_{\theta}$. The second question concerns the behavior of the solution as $\theta \rightarrow 0$. This problem is important from the mechanical point of view since it concerns the behavior of the solution of a frictional viscoelastic contact problem as the viscosity goes to zero. Clearly, if we take $\theta=0$, the first term on the left hand side of (1.1) vanishes. Therefore it is natural to consider the inviscid problem associated to (1.1)-(1.2), that is:

Problem $P$. Find a function $u:[0, T] \rightarrow V$ such that

$$
\begin{aligned}
(B u(t), v-\dot{u}(t))_{V} & +j(v)-j(\dot{u}(t)) \\
& \geq(f(t), v-\dot{u}(t))_{V} \quad \forall v \in V, \text { a.e. } t \in(0, T), \\
u(0) & =u_{0} .
\end{aligned}
$$

Problems of this form arise in the study of quasistatic frictional models with elastic materials (see for instance $[4,9]$ ). Existence and uniqueness results for abstract evolutionary inequalities of the form (1.3)-(1.4) can be found in $[1,2,9]$.

The aim of this paper is to study the behavior of the solution of Problem $P_{\theta}$ as $\theta \rightarrow 0$ with emphasis on problems arising in Contact Mechanics and more precisely on antiplane frictional contact problems. Various results on quasistatic antiplane contact problems, including the existence of weak solutions, were obtained in $[5,8,11]$. In [5] the material was assumed to have a viscoelastic behavior while in [8] it was assumed to be linearly elastic; in both these papers the contact was frictional and it was modelled by a slip-dependent friction law. In [11] the material was assumed to be elastic and the contact was frictionless but adhesive.

The paper is organized as follows. In Section 2 we state two existence and uniqueness results for Problems $P_{\theta}$ and $P$, respectively (Theorems 2.1 and 2.2). In Section 3 we prove that the solution $u_{\theta}$ of Problem $P_{\theta}$ converges to the solution $u$ of Problem $P$ as $\theta \rightarrow 0$ (Theorem 3.1). In Section 4 we 
present mathematical models which describe the antiplane frictional contact problem with elastic and viscoelastic materials. Finally, in Section 5 we derive the variational formulation of the models and we apply the abstract results of Sections 2 and 3 to obtain existence, uniqueness and convergence results for antiplane problems (Theorem 5.1).

2. Existence and uniqueness results. We use the standard notation for $L^{p}(0, T ; V)$ and Sobolev spaces $W^{k, p}(0, T ; V), k \in \mathbb{N}, 1 \leq p \leq \infty$. We also denote by $C([0, T] ; V)$ the space of continuous functions from $[0, T]$ to $V$, with norm

$$
\|u\|_{C([0, T] ; V)}=\max _{t \in[0, T]}\|u(t)\|_{V} .
$$

We assume that $A: V \rightarrow V$ is a strongly monotone Lipschitz continuous operator, i.e.,

$$
\left\{\begin{array}{l}
\text { (a) there exists } m>0 \text { such that } \\
\left(A u_{1}-A u_{2}, u_{1}-u_{2}\right)_{V} \geq m\left\|u_{1}-u_{2}\right\|_{V}^{2} \quad \forall u_{1}, u_{2} \in V ; \\
\text { (b) there exists } M>0 \text { such that } \\
\\
\left\|A u_{1}-A u_{2}\right\|_{V} \leq M\left\|u_{1}-u_{2}\right\|_{V} \quad \forall u_{1}, u_{2} \in V .
\end{array}\right.
$$

The operator $B: V \rightarrow V$ is symmetric, continuous and positive definite, that is,

$$
\left\{\begin{array}{l}
\text { (a) }(B u, v)_{V}=(u, B v)_{V} \quad \forall u, v \in V \\
\text { (b) there exists } M^{\prime}>0 \text { such that }\|B u\|_{V} \leq M^{\prime}\|u\|_{V} \forall u \in V ; \\
\text { (c) there exists } m^{\prime}>0 \text { such that }(B u, u)_{V} \geq m^{\prime}\|u\|_{V}^{2} \forall u \in V .
\end{array}\right.
$$

Finally, about the data $j, f$ and $u_{0}$ we assume that:

$$
\begin{gathered}
j: V \rightarrow \mathbb{R} \text { is convex and lower semicontinuous, } \\
f \in W^{1,2}(0, T ; V), \\
u_{0} \in V, \\
\sup _{v \in V}\left\{(f(0), v)_{V}-\left(B u_{0}, v\right)_{V}-j(v)\right\}<\infty .
\end{gathered}
$$

The unique solvability of Problems $P_{\theta}$ and $P$ is the content of the following results.

Theorem 2.1. Let (2.1)-(2.5) hold. Then there exists a unique solution $u \in W^{1,2}(0, T ; V)$ to problem $(1.1)-(1.2)$.

TheOREM 2.2. Let (2.2)-(2.6) hold. Then there exists a unique solution $u \in W^{1,2}(0, T ; V)$ to problem $(1.3)-(1.4)$. 
Both theorems are versions of results which can be found in the literature and therefore we do not provide their proofs. Indeed, Theorem 2.1 represents a simplified version of a more general existence and uniqueness result obtained in [3]. There, it was assumed that $A$ satisfies (2.1), $B$ is a nonlinear Lipschitz continuous operator on $V$ and the functional $j$ depends on the solution, i.e. $j=j(u, v)$. The proof, based on arguments of elliptic variational inequalities and fixed point theory, can also be found in $[4$, pp. 230-234]. Theorem 2.2 is a version of a result proved in [1, p. 117] by arguments of nonlinear evolution equations with maximal monotone operators; there, $j$ was assumed to be a proper, convex and lower semicontinous function on $V$ with values in $]-\infty, \infty]$ and $B$ was the identity operator on $V$. A variant of Theorem 2.2 is given in [2, p. 158], where the proof is based on a time-discretization method together with compactness and lower semicontinuity arguments; in [2], $j$ was assumed to be a convex, positively homogeneous and Lipschitz continuous real-valued function. A version of Theorem 2.2 in the case when $j$ depends on the solution, i.e. $j=j(u, v)$, was established in [9]; the conditions imposed there on the nondifferentiable functional $j$ were formulated in terms of the directional derivative. Both in [2] and [9] the operator $B$ was assumed to satisfy condition (2.2).

3. A convergence result. We now study the behavior of the solution $u_{\theta}$ of Problem $P_{\theta}$ as $\theta \rightarrow 0$. The main result of this section is the following.

Theorem 3.1. Assume (2.1)-(2.6) hold and denote by $u_{\theta}$ and $u$ the solutions of Problems $P_{\theta}$ and $P$, guaranteed by Theorems 2.1 and 2.2, respectively. Then

$$
\left(\dot{u}_{\theta}\right) \text { is a bounded sequence in } L^{2}(0, T ; V) \text { as } \theta \rightarrow 0 \text {; }
$$

$$
u_{\theta} \rightarrow u \quad \text { in } C([0, T], V) \text { as } \theta \rightarrow 0 .
$$

Proof. The equalities and inequalities below hold for almost all $t \in$ $(0, T)$. We take $v=\dot{u}(t)$ in $(1.1), v=\dot{u}_{\theta}(t)$ in (1.3) and add the corresponding inequalities to obtain

$$
\theta\left(A \dot{u}_{\theta}(t), \dot{u}(t)-\dot{u}_{\theta}(t)\right)_{V}+\left(B u_{\theta}(t)-B u, \dot{u}(t)-\dot{u}_{\theta}(t)\right)_{V} \geq 0 .
$$

This implies

$$
\begin{array}{r}
\theta\left(A \dot{u}_{\theta}(t)-A \dot{u}(t), \dot{u}(t)-\dot{u}_{\theta}(t)\right)_{V}+\theta\left(A \dot{u}(t), \dot{u}(t)-\dot{u}_{\theta}(t)\right)_{V} \\
+\left(B u_{\theta}(t)-B u(t), \dot{u}(t)-\dot{u}_{\theta}(t)\right)_{V} \geq 0
\end{array}
$$

and, using (2.1), yields

$$
\begin{aligned}
& \theta m\left\|\dot{u}_{\theta}(t)-\dot{u}(t)\right\|_{V}^{2}+\left(B u_{\theta}(t)-B u(t), \dot{u}_{\theta}(t)-\dot{u}(t)\right)_{V} \\
& \quad \leq \theta M\|\dot{u}(t)\|_{V}\left\|\dot{u}_{\theta}(t)-\dot{u}(t)\right\|_{V}+\theta\left\|A 0_{V}\right\|_{V}\left\|\dot{u}_{\theta}(t)-\dot{u}(t)\right\|_{V} .
\end{aligned}
$$

Here and below $0_{V}$ will represent the zero element of the space $V$. 
Let $s \in[0, T]$ be given. We integrate (3.3) on $[0, s]$ and use the properties (2.2) of $B$ as well as the initial conditions (1.2) and (1.4) to find

$$
\begin{aligned}
& \theta m \int_{0}^{s}\left\|\dot{u}_{\theta}(t)-\dot{u}(t)\right\|_{V}^{2} d t+\frac{1}{2}\left(B u_{\theta}(t)-B u(t), u_{\theta}(t)-u(t)\right)_{V} \\
& \leq \theta M \int_{0}^{s}\|\dot{u}(t)\|_{V}\left\|\dot{u}_{\theta}(t)-\dot{u}(t)\right\|_{V} d t+\theta \int_{0}^{s}\left\|A 0_{V}\right\|_{V}\left\|\dot{u}_{\theta}(t)-\dot{u}(t)\right\|_{V} d t .
\end{aligned}
$$

We now apply the inequalities

$$
\begin{aligned}
\|\dot{u}(t)\|_{V}\left\|\dot{u}_{\theta}(t)-\dot{u}(t)\right\|_{V} & \leq \frac{M}{2 m}\|\dot{u}(t)\|_{V}^{2}+\frac{m}{2 M}\left\|\dot{u}_{\theta}(t)-\dot{u}(t)\right\|_{V}^{2}, \\
\left\|A 0_{V}\right\|_{V}\left\|\dot{u}_{\theta}(t)-\dot{u}(t)\right\|_{V} & \leq \frac{M}{2 m}\left\|A 0_{V}\right\|_{V}^{2}+\frac{m}{2 M}\left\|\dot{u}_{\theta}(t)-\dot{u}(t)\right\|_{V}^{2}
\end{aligned}
$$

in (3.4) to obtain

$$
\begin{aligned}
\theta m \int_{0}^{s}\left\|\dot{u}_{\theta}(t)-\dot{u}(t)\right\|_{V}^{2} d t+\frac{1}{2}\left(B u_{\theta}(s)-B u(s), u_{\theta}(s)-u(s)\right)_{V} \\
\leq \leq \frac{\theta M^{2}}{2 m} \int_{0}^{s}\|\dot{u}(t)\|_{V}^{2} d t \\
\quad+\frac{\theta M T}{2 m}\left\|A 0_{V}\right\|_{V}^{2}+\frac{\theta m}{2}\left(1+\frac{1}{M}\right) \int_{0}^{s}\left\|\dot{u}_{\theta}(t)-\dot{u}(t)\right\|_{V}^{2} d t
\end{aligned}
$$

Since $\left(B u_{\theta}(s)-B u(s), u_{\theta}(s)-u(s)\right)_{V} \geq 0$, from (3.5) we deduce that

$$
\frac{\theta m}{2}\left(1-\frac{1}{M}\right) \int_{0}^{s}\left\|\dot{u}_{\theta}(t)-\dot{u}(t)\right\|_{V}^{2} d t \leq \frac{\theta M^{2}}{2 m} \int_{0}^{s}\|\dot{u}(t)\|_{V}^{2} d t+\frac{\theta M T}{2 m}\left\|A 0_{V}\right\|_{V}^{2} .
$$

We now take $s=T$ to obtain

$$
\frac{\theta m}{2}\left(1-\frac{1}{M}\right)\left\|\dot{u}_{\theta}-\dot{u}\right\|_{L^{2}(0, T ; V)}^{2} \leq \frac{\theta M^{2}}{2 m}\|\dot{u}\|_{L^{2}(0, T ; V)}^{2}+\frac{\theta M T}{2 m}\left\|A 0_{V}\right\|_{V}^{2} .
$$

Since we can assume that $M>1$, we find that

$$
\left\|\dot{u}_{\theta}-\dot{u}\right\|_{L^{2}(0, T ; V)} \quad \text { is bounded as } \theta \rightarrow 0 .
$$

The boundedness result (3.1) is now a consequence of (3.6).

We use again (3.5) and (2.2)(c) to find

$$
\begin{aligned}
\frac{m^{\prime}}{2}\left\|u_{\theta}(s)-u(s)\right\|_{V}^{2} \leq & \frac{\theta M^{2}}{2 m} \int_{0}^{s}\|\dot{u}(t)\|_{V}^{2} d t+\frac{\theta M T}{2 m}\left\|A 0_{V}\right\|_{V}^{2} \\
& +\frac{\theta m}{2}\left(1+\frac{1}{M}\right) \int_{0}^{s}\left\|\dot{u}_{\theta}(t)-\dot{u}(t)\right\|_{V}^{2} d t
\end{aligned}
$$


which implies that

$$
\begin{aligned}
\frac{m^{\prime}}{2}\left\|u_{\theta}(s)-u(s)\right\|_{V}^{2} \leq & \frac{\theta M^{2}}{2 m}\|\dot{u}\|_{L^{2}(0, T ; V)}^{2}+\frac{\theta M T}{2 m}\left\|A 0_{V}\right\|_{V}^{2} \\
& +\frac{\theta m}{2}\left(1+\frac{1}{M}\right)\left\|\dot{u}_{\theta}-\dot{u}\right\|_{L^{2}(0, T ; V)}^{2}
\end{aligned}
$$

The convergence result (3.2) is now a consequence of (3.6) and (3.7).

4. A model of antiplane frictional contact problems. The abstract results of Theorems 2.1, 2.2 and 3.1 are useful in the study of a wide class of problems arising in Contact Mechanics. To provide an example, we present a model of antiplane contact problems involving viscoelastic and elastic materials. Everywhere below we use bold face symbols to denote vectors and tensors. Moreover, we use "." for the inner product in the spaces $\mathbb{R}^{2}$ and $\mathbb{R}^{3}$, and $|\cdot|$ for the Euclidean norm.

We consider a body $\mathcal{B}$ identified with a region in $\mathbb{R}^{3}$ it occupies in a fixed and undistorted reference configuration. We assume that $\mathcal{B}$ is a cylinder with generators parallel to the $x_{3}$-axis with a cross-section which is a regular region $\Omega$ in the $x_{1}, x_{2}$-plane, $O x_{1} x_{2} x_{3}$ being a Cartesian coordinate system. The cylinder is assumed to be sufficiently long so that the end effects in the axial direction are negligible. Thus, $\mathcal{B}=\Omega \times(-\infty, \infty)$. Let $\partial \Omega=\Gamma$. We assume that $\Gamma$ is divided into three disjoint measurable parts $\Gamma_{1}, \Gamma_{2}$ and $\Gamma_{3}$ such that the one-dimensional measure of $\Gamma_{1}$, denoted by meas $\Gamma_{1}$, is strictly positive. Let $T>0$ and let $[0, T]$ be the time interval of interest. The cylinder is clamped on $\Gamma_{1} \times(-\infty, \infty)$ and is in contact with a rigid foundation on $\Gamma_{3} \times(-\infty, \infty)$ during the process. Moreover, the cylinder is subjected to time dependent volume forces of density $\boldsymbol{f}_{0}$ on $\mathcal{B}$ and to time dependent surface tractions of density $\boldsymbol{f}_{2}$ on $\Gamma_{2} \times(-\infty, \infty)$.

We assume that

$$
\begin{aligned}
& \boldsymbol{f}_{0}=\left(0,0, f_{0}\right) \quad \text { with } \quad f_{0}=f_{0}\left(x_{1}, x_{2}, t\right): \Omega \times[0, T] \rightarrow \mathbb{R}, \\
& \boldsymbol{f}_{2}=\left(0,0, f_{2}\right) \quad \text { with } \quad f_{2}=f_{2}\left(x_{1}, x_{2}, t\right): \Gamma_{2} \times[0, T] \rightarrow \mathbb{R} .
\end{aligned}
$$

The body forces (4.1) and the surface tractions (4.2) are expected to give rise to a deformation of the cylinder whose displacement, denoted by $\boldsymbol{u}$, is independent of $x_{3}$ and has the form

$$
\boldsymbol{u}=(0,0, u) \quad \text { with } \quad u=u\left(x_{1}, x_{2}, t\right): \Omega \times[0, T] \rightarrow \mathbb{R} .
$$

Such kind of deformation is called an antiplane shear; see for instance $[7,6]$ for details.

Let $\boldsymbol{\varepsilon}(\boldsymbol{u})=\left(\varepsilon_{i j}(\boldsymbol{u})\right)$ be the infinitesimal strain tensor, that is,

$$
\varepsilon_{i j}(\boldsymbol{u})=\frac{1}{2}\left(u_{i, j}+u_{j, i}\right),
$$


where the indices $i$ and $j$ run from 1 to 3 and the index that follows a comma indicates a partial derivative with respect to the corresponding component of the spatial variable. Let also $\boldsymbol{\sigma}=\left(\boldsymbol{\sigma}_{i j}\right)$ denote the stress field. We assume that the material is modelled by the following linear viscoelastic constitutive law with short memory:

$$
\boldsymbol{\sigma}=2 \theta \varepsilon(\dot{\boldsymbol{u}})+\lambda(\operatorname{tr} \boldsymbol{\varepsilon}(\boldsymbol{u})) \boldsymbol{I}+2 \mu \boldsymbol{\varepsilon}(\boldsymbol{u}),
$$

where $\theta>0$ is the coefficient of viscosity, $\lambda, \mu>0$ are the Lamé coefficients, $\operatorname{tr} \varepsilon(\boldsymbol{u})=\varepsilon_{i i}(\boldsymbol{u})$, and $\boldsymbol{I}$ is the unit tensor in $\mathbb{R}^{3}$. Here and below, to simplify the notation, we do not indicate the dependence of various functions on $x_{1}$, $x_{2}$ or $t$. Moreover, the convention summation upon repeated indices is used.

It follows from (4.3)-(4.5) that

$$
\begin{aligned}
& \sigma_{11}=\sigma_{12}=\sigma_{21}=\sigma_{22}=\sigma_{33}=0, \\
& \sigma_{3 i}=\sigma_{i 3}=\theta \dot{u}_{, i}+\mu u_{, i} \quad \forall i=1,2 .
\end{aligned}
$$

Neglecting the inertial term in the equation of motion we obtain the quasistatic approximation for the process. Thus, by (4.6), the equation of equilibrium reduces to the scalar equation

$$
\theta \Delta \dot{u}+\mu \Delta u+f_{0}=0 \quad \text { in } \Omega \times(0, T) .
$$

As the cylinder is clamped on $\Gamma_{1} \times(-\infty, \infty) \times(0, T)$, the displacement field vanishes there. Therefore, (4.3) implies that

$$
u=0 \quad \text { on } \Gamma_{1} \times(0, T) .
$$

Let $\boldsymbol{\nu}$ denote the unit normal on $\Gamma \times(-\infty, \infty)$. We have

$$
\boldsymbol{\nu}=\left(\nu_{1}, \nu_{2}, 0\right) \quad \text { with } \quad \nu_{i}=\nu_{i}\left(x_{1}, x_{2}\right): \Gamma \rightarrow \mathbb{R}, i=1,2 .
$$

For a vector $\boldsymbol{v}$ we denote by $v_{\nu}$ and $\boldsymbol{v}_{\tau}$ its normal and tangential components on the boundary, given by

$$
v_{\nu}=\boldsymbol{v} \cdot \boldsymbol{\nu}, \quad \boldsymbol{v}_{\tau}=\boldsymbol{v}-v_{\nu} \boldsymbol{\nu}
$$

For a given stress field $\boldsymbol{\sigma}$ we also denote by $\sigma_{\nu}$ and $\boldsymbol{\sigma}_{\tau}$ its normal and tangential components on the boundary, that is,

$$
\sigma_{\nu}=(\boldsymbol{\sigma} \boldsymbol{\nu}) \cdot \boldsymbol{\nu}, \quad \boldsymbol{\sigma}_{\tau}=\boldsymbol{\sigma} \boldsymbol{\nu}-\sigma_{\nu} \boldsymbol{\nu} .
$$

From (4.3), (4.6), (4.7) and (4.9) we deduce that the Cauchy stress vector is given by

$$
\boldsymbol{\sigma} \boldsymbol{\nu}=\left(0,0, \theta \partial_{\nu} \dot{u}+\mu \partial_{\nu} u\right)
$$

Here and below we use the notation $\partial_{\nu} u=u_{, 1} \nu_{1}+u_{, 2} \nu_{2}$. Together with the traction boundary condition $\boldsymbol{\sigma} \boldsymbol{\nu}=\boldsymbol{f}_{2}$ on $\Gamma_{2} \times(0, T)$, it follows from (4.2) and (4.10) that

$$
\theta \partial_{\nu} \dot{u}+\mu \partial_{\nu} u=f_{2} \quad \text { on } \Gamma_{2} \times(0, T) .
$$


We now describe the contact condition on $\Gamma_{3} \times(-\infty, \infty)$. First, from (4.3) and (4.8) we infer that $u_{\nu}=0$, which shows that the contact is bilateral, that is, it is kept during all the process. Using now (4.3), (4.8)-(4.10) we conclude that

$$
\boldsymbol{u}_{\tau}=(0,0, u), \quad \boldsymbol{\sigma}_{\tau}=\left(0,0, \theta \partial_{\nu} \dot{u}+\mu \partial_{\nu} u\right) .
$$

We assume that when slip arises, then the tangential shear is proportional to the $p$ th power of the tangential speed, that is,

$$
\dot{\boldsymbol{u}}_{\tau} \neq \mathbf{0} \Rightarrow-\boldsymbol{\sigma}_{\tau}=\kappa\left|\dot{\boldsymbol{u}}_{\tau}\right|^{p-1} \dot{\boldsymbol{u}}_{\tau} \quad \text { on } \Gamma_{3} \times(0, T) .
$$

Such a frictional boundary condition arises when the contact surface is lubricated with a thin layer of a non-Newtonian fluid and was already considered in $[10,12]$. Here $\kappa: \Gamma_{3} \rightarrow \mathbb{R}_{+}$is a positive function, $\dot{\boldsymbol{u}}_{\tau}$ represents the tangential velocity on the contact boundary and $0<p \leq 1$. Using now (4.11) it is straightforward to see that the condition (4.12) implies

$$
\dot{u} \neq 0 \Rightarrow \theta \partial_{\nu} \dot{u}+\mu \partial_{\nu} u=-\kappa\left|\dot{u}_{\tau}\right|^{p-1} \dot{u}_{\tau} \quad \text { on } \Gamma_{3} \times(0, T) .
$$

Finally, we prescribe the initial displacement,

$$
u(0)=u_{0} \quad \text { in } \Omega,
$$

where $u_{0}$ is a given function on $\Omega$.

We collect the above equations and conditions to obtain the classical formulation of the antiplane problem for viscoelastic materials in frictional contact with a foundation. The subscript $\theta$ indicating the dependence of the solution on the coefficient of viscosity, the formulation is the following.

Problem $\mathcal{P}_{\theta}$. Find a displacement field $u_{\theta}: \Omega \times[0, T] \rightarrow \mathbb{R}$ such that

$$
\begin{aligned}
& \theta \Delta \dot{u}_{\theta}+\mu \Delta u_{\theta}+f_{0}=0 \quad \text { in } \Omega \times(0, T), \\
& u_{\theta}=0 \quad \text { on } \Gamma_{1} \times(0, T) \text {, } \\
& \theta \partial_{\nu} \dot{u}_{\theta}+\mu \partial_{\nu} u_{\theta}=f_{2} \quad \text { on } \Gamma_{2} \times(0, T), \\
& \dot{u}_{\theta} \neq 0 \Rightarrow \theta \partial_{\nu} \dot{u}_{\theta}+\mu \partial_{\nu} u_{\theta}=-\kappa\left|\dot{u}_{\theta \tau}\right|^{p-1} \dot{u}_{\theta \tau} \quad \text { on } \Gamma_{3} \times(0, T) \text {, } \\
& u_{\theta}(0)=u_{0} \quad \text { in } \Omega .
\end{aligned}
$$

Notice that once the displacement field $u_{\theta}$ which solves Problem $\mathcal{P}_{\theta}$ is known, the associated stress tensor, denoted by $\boldsymbol{\sigma}^{\theta}=\left(\sigma_{i j}^{\theta}\right)$, can be calculated using formulas (4.6) in which $u$ is replaced by $u_{\theta}$. We obtain

$$
\begin{aligned}
& \sigma_{11}^{\theta}=\sigma_{12}^{\theta}=\sigma_{21}^{\theta}=\sigma_{22}^{\theta}=\sigma_{33}^{\theta}=0, \\
& \sigma_{3 i}^{\theta}=\sigma_{i 3}^{\theta}=\theta \dot{u}_{\theta, i}+\mu u_{\theta, i} \forall i=1,2 .
\end{aligned}
$$

The inviscid problem is obtained for $\theta=0$ and can be formulated as follows. 
Problem $\mathcal{P}$. Find a displacement field $u: \Omega \times[0, T] \rightarrow \mathbb{R}$ such that

$$
\begin{array}{cl}
\mu \Delta u+f_{0}=0 \quad & \text { in } \Omega \times(0, T), \\
u=0 \quad & \text { on } \Gamma_{1} \times(0, T), \\
\mu \partial_{\nu} u=f_{2} \quad & \text { on } \Gamma_{2} \times(0, T), \\
\dot{u} \neq 0 \Rightarrow \mu \partial_{\nu} u=-\mu\left|\dot{u}_{\tau}\right|^{p-1} \dot{u}_{\tau} \quad \text { on } \Gamma_{3} \times(0, T), & \\
u(0)=u_{0} \quad & \text { in } \Omega .
\end{array}
$$

Clearly, problem $\mathcal{P}$ represents the variational formulation of the antiplane frictional contact problem for linear elastic materials, i.e. the problem obtained when (4.5) is replaced by the elastic constitutive law

$$
\boldsymbol{\sigma}=\lambda(\operatorname{tr}(\boldsymbol{\varepsilon}(\boldsymbol{u}))) \boldsymbol{I}+2 \mu \boldsymbol{\varepsilon}(\boldsymbol{u})
$$

Notice that in this case, taking $\theta=0$ in (4.6), we obtain

$$
\begin{aligned}
& \sigma_{11}=\sigma_{12}=\sigma_{21}=\sigma_{22}=\sigma_{33}=0, \\
& \sigma_{3 i}=\sigma_{i 3}=\mu u_{, i} \quad \forall i=1,2 .
\end{aligned}
$$

The mathematical analysis of the antiplane contact problems $\mathcal{P}_{\theta}$ and $\mathcal{P}$ will be provided in the next section.

5. Existence, uniqueness and convergence results. We now derive the variational formulation of Problems $\mathcal{P}_{\theta}$ and $\mathcal{P}$. We show that these formulations are of the form (1.1)-(1.2), (1.3)-(1.4), respectively, and then we apply the abstract results of Theorems 2.1, 2.2 and 3.1.

To this end, in this section we shall specify the space $V$ as follows:

$$
V=\left\{v \in H^{1}(\Omega) \mid v=0 \text { on } \Gamma_{1}\right\} .
$$

Here and below we write $v$ for the trace $\gamma v$ of $v$ on $\Gamma$. Moreover, we use $\nabla$ for the gradient operator on $\Omega$, i.e. $\nabla v=\left(v_{, 1}, v_{, 2}\right)$.

On $V$ we consider the inner product of the Sobolev space $H^{1}(\Omega)$, that is,

$$
(u, v)_{V}=\int_{\Omega} u v d x+\int_{\Omega} \nabla u \cdot \nabla v d x \quad \forall u, v \in V,
$$

and let $\|\cdot\|_{V}$ be the associated norm, i.e.

$$
\|v\|_{V}^{2}=\int_{\Omega}|v|^{2} d x+\int_{\Omega}|\nabla v|^{2} d x \quad \forall v \in V .
$$

Notice that $V$ is a closed subspace of $H^{1}(\Omega)$ and therefore $\left(V,\|\cdot\|_{V}\right)$ is a real Hilbert space.

In the study of the mechanical problems $\mathcal{P}_{\theta}$ and $\mathcal{P}$, we assume that the forces and tractions have the regularity

$$
f_{0} \in W^{1,2}\left(0, T ; L^{2}(\Omega)\right), \quad f_{2} \in W^{1,2}\left(0, T ; L^{2}\left(\Gamma_{2}\right)\right),
$$


the function $\kappa$ satisfies

$$
\kappa \in L^{\infty}\left(\Gamma_{3}\right), \quad \kappa(x) \geq 0 \quad \text { a.e. } x \in \Gamma_{3},
$$

and the initial data satisfies

$$
u_{0} \in V \text {. }
$$

In the study of Problem $\mathcal{P}$ we also assume that

$$
\begin{aligned}
\mu \int_{\Omega} \nabla u_{0} \cdot \nabla v d x+\frac{1}{p+1} \int_{\Gamma_{3}} & \kappa|v|^{p+1} d a \\
& \geq \int_{\Omega} f_{0}(0) v d x+\int_{\Gamma_{2}} f_{2}(0) v d a \quad \forall v \in V .
\end{aligned}
$$

Notice that inequality (5.5) represents a compatibility condition on the initial and boundary data which, physically, guarantees that the initial state of the elastic body is an equilibrium one.

Using Green's formula and the inequality

$$
|a|^{p-1} a(b-a) \leq \frac{1}{p+1}|b|^{p+1}-\frac{1}{p+1}|a|^{p+1} \quad \forall a, b \in \mathbb{R}, a \neq 0,
$$

it is straightforward to derive the following variational formulation of Problem $\mathcal{P}_{\theta}$.

Problem $\mathcal{P}_{\theta}^{V}$. Find a displacement field $u_{\theta}:[0, T] \rightarrow V$ such that

$$
\begin{array}{r}
\theta \int_{\Omega} \nabla u_{\theta}(t) \cdot\left(\nabla v-\nabla \dot{u}_{\theta}(t)\right) d x+\mu \int_{\Omega} \nabla u_{\theta}(t) \cdot\left(\nabla v-\nabla \dot{u}_{\theta}(t)\right) d x \\
+\frac{1}{p+1} \int_{\Gamma_{3}} \kappa|v|^{p+1} d a-\frac{1}{p+1} \int_{\Gamma_{3}} \kappa\left|\dot{u}_{\theta}(t)\right|^{p+1} d a \\
\geq \int_{\Omega} f_{0}(t)\left(v-\dot{u}_{\theta}(t)\right) d x+\int_{\Gamma_{2}} f_{2}(t)\left(v-\dot{u}_{\theta}(t)\right) d a \\
\forall v \in V, \text { a.e. } t \in(0, T),
\end{array}
$$

Alternatively, the variational formulation of Problem $\mathcal{P}$ is the following.

Problem $\mathcal{P}^{V}$. Find a displacement field $u:[0, T] \rightarrow V$ such that

$$
\begin{aligned}
\mu \int_{\Omega} \nabla u(t) \cdot(\nabla v-\nabla \dot{u}(t)) d x & \\
+\frac{1}{p+1} \int_{\Gamma_{3}} \kappa|v|^{p+1} d a-\frac{1}{p+1} \int_{\Gamma_{3}} \kappa|\dot{u}(t)|^{p+1} d a & \\
& \geq \int_{\Omega} f_{0}(t)(v-\dot{u}(t)) d x+\int_{\Gamma_{2}} f_{2}(t)(v-\dot{u}(t)) d a \\
& \forall v \in V, \text { a.e. } t \in(0, T), \\
u(0) & =u_{0} .
\end{aligned}
$$


Our main result in this section is the following.

THEOREM 5.1.

(a) Under the assumptions (5.2)-(5.4) there exists a unique solution $u_{\theta} \in$ $W^{1,2}(0, T ; V)$ to problem $\mathcal{P}_{\theta}^{V}$.

(b) Under the assumptions (5.2)-(5.5) there exists a unique solution $u \in$ $W^{1,2}(0, T ; V)$ to problem $\mathcal{P}^{V}$.

(c) Let (5.2)-(5.5) hold, denote by $u_{\theta}$ and $u$ the solutions obtained above, and let $\sigma_{i j}^{\theta}$ and $\sigma_{i j}$ be the components of the corresponding stress fields given by (4.18) and (4.24), respectively. Then

$$
\begin{gathered}
u_{\theta} \rightarrow u \quad \text { in } C([0, T] ; V) \text { as } \theta \rightarrow 0, \\
\sigma_{i j}^{\theta} \rightarrow \sigma_{i j} \quad \text { in } L^{2}\left(0, T ; L^{2}(\Omega)\right) \text { as } \theta \rightarrow 0, \text { for all } i, j=1,2,3 .
\end{gathered}
$$

Proof. (a) We use Riesz's representation theorem to define the operators $A, B: V \rightarrow V$, the functional $j: V \rightarrow \mathbb{R}_{+}$and the function $f:[0, T] \rightarrow V$ by the following equalities:

$$
\begin{array}{cl}
(A u, v)_{V}=\int_{\Gamma_{3}} \nabla u \cdot \nabla v d a & \forall u, v \in V, \\
(B u, v)_{V}=\mu \int_{\Gamma_{3}} \nabla u \cdot \nabla v d a & \forall u, v \in V, \\
j(v)=\frac{1}{p+1} \int_{\Gamma_{3}} \kappa|v|^{p+1} d a & \forall v \in V, \\
(f(t), v)_{V}=\int_{\Omega} f_{0}(t) v d x+\int_{\Gamma_{2}} f_{2}(t) v d a & \forall v \in V, t \in[0, T] .
\end{array}
$$

Since meas $\Gamma_{1}>0$, the Friedrichs-Poincaré inequality holds, that is, there exists a positive constant $C_{P}$ (which depends only on $\Omega$ and $\Gamma_{1}$ ) such that

$$
\|u\|_{V}^{2} \leq C_{P} \int_{\Omega}|\nabla u|^{2} d x \quad \forall u \in V .
$$

Using (5.1) and (5.16) it is easy to see that the operators $A$ and $B$ satisfy conditions (2.1) and (2.2). Clearly the functional $j$ defined by (5.15) is convex. Moreover, by Sobolev's trace theorem we deduce that there exists $C_{0}>0$ (depending only on $\Omega$ and $\Gamma$ ) such that

$$
\left(\int_{\Gamma}|v|^{2} d a\right)^{1 / 2} \leq C_{0}\|v\|_{V} \quad \forall v \in V .
$$

Therefore (5.3) implies that $j$ is continuous and hence it satisfies condition (2.3). Also, from (5.2) it follows that $f$ satisfies condition (2.4) and, in view of (5.4), it follows that (2.5) also holds. The existence of a unique solution 
$u_{\theta} \in W^{1,2}(0, T ; V)$ of Problem $\mathcal{P}_{\theta}^{V}$ is now a consequence of Theorem 2.1 and equalities (5.12)-(5.15).

(b) It follows from (5.13)-(5.15) and (5.5) that

$$
\left(B u_{0}, v\right)_{V}+j(v) \geq(f(0), v)_{V} \quad \forall v \in V,
$$

and therefore condition (2.6) is satisfied. The existence of a unique solution $u \in W^{1,2}(0, T ; V)$ of Problem $\mathcal{P}^{V}$ is now a consequence of Theorem 2.2 and equalities (5.12)-(5.15).

(c) The convergence result (5.10) follows from the second part of Theorem 3.1 (see (3.2)). Next, we recall that from (4.18) and (4.24) we have to prove (5.11) only for $i=1,2$ and $j=3$. Let $i \in\{1,2\}$. Then

$$
\sigma_{3 i}^{\theta}=\sigma_{i 3}^{\theta}=\theta \dot{u}_{\theta, i}+\mu u_{\theta, i}, \quad \sigma_{3 i}=\sigma_{i 3}=\mu u_{, i},
$$

and since $u_{\theta}, u \in W^{1,2}(0, T ; V)$, it follows that $\sigma_{i 3}^{\theta} \in L^{2}\left(0, T ; L^{2}(\Omega)\right)$ and $\sigma_{i 3} \in W^{1,2}\left(0, T ; L^{2}(\Omega)\right)$. Moreover,

$$
\begin{aligned}
& \left\|\sigma_{i 3}^{\theta}(t)-\sigma_{i 3}(t)\right\|_{L^{2}(\Omega)}^{2}=\theta \int_{\Omega}\left|\dot{u}_{\theta, i}(t)\right|^{2} d x+\mu \int_{\Omega}\left|u_{\theta, i}(t)-u_{, i}(t)\right|^{2} d x \\
& \leq \theta \int_{\Omega}|\nabla \dot{u}(t)|^{2} d x+\mu \int_{\Omega}\left|\nabla u_{\theta}(t)-\nabla u(t)\right|^{2} d x \quad \text { a.e. } t \in(0, T) .
\end{aligned}
$$

By (5.1) we obtain

$$
\int_{0}^{T}\left\|\sigma_{i 3}^{\theta}(t)-\sigma_{i 3}(t)\right\|_{L^{2}(\Omega)}^{2} d t \leq \theta \int_{0}^{T}\left\|\dot{u}_{\theta}\right\|_{V}^{2} d t+\mu \int_{0}^{T}\left\|u_{\theta}(t)-u(t)\right\|_{V}^{2} d t .
$$

We use again (3.1) and (3.2) to deduce that

$$
\int_{0}^{T}\left\|\sigma_{i 3}^{\theta}(t)-\sigma_{i 3}(t)\right\|_{L^{2}(\Omega)}^{2} d t \rightarrow 0 \quad \text { as } \theta \rightarrow 0,
$$

which shows (5.11) for $i=1,2$ and $j=3$, concluding the proof.

We now provide a mechanical interpretation of Theorem 5.1.

An element $u_{\theta}$ which solves Problem $\mathcal{P}_{\theta}^{V}$ is called a weak solution of the viscoelastic problem $\mathcal{P}_{\theta}$, while $u$ solving Problem $\mathcal{P}^{V}$ is a weak solution of the elastic problem $\mathcal{P}$. We conclude by Theorem $5.1(\mathrm{a}, \mathrm{b})$ that the antiplane contact problems $\mathcal{P}_{\theta}$ and $\mathcal{P}$ have unique weak solutions. Also, from Theorem 5.1(c) we conclude that the weak solution to the antiplane elastic problem with friction may be approached by the weak solution to the antiplane viscoelastic problem with friction as the viscosity is small enough. Besides the mathematical interest of this result, it is of importance from the mechanical point of view, as it indicates that elasticity with friction may be considered as a limit case of viscoelasticity with friction as the viscosity decreases. 


\section{References}

[1] H. Brezis, Problèmes unilatéraux, J. Math. Pures Appl. 51 (1972), 1-168.

[2] W. Han and B. D. Reddy, Plasticity: Mathematical Theory and Numerical Analysis, Springer, New York, 1999.

[3] W. Han and M. Sofonea, Time-dependent variational inequalities for viscoelastic contact problems, J. Comput. Appl. Math. 136 (2001), 369-387.

[4] -, -, Quasistatic Contact Problems in Viscoelasticity and Viscoplasticity, Stud. Adv. Math. 30, Amer. Math. Soc.-Intl. Press, 2002.

[5] T.-V. Hoarau-Mantel and A. Matei, Analysis of a viscoelastic antiplane contact problem with slip-dependent friction, Int. J. Appl. Math. Comput. Sci. 12 (2002), $51-58$.

[6] C. O. Horgan, Anti-plane shear deformation in linear and nonlinear solid mechanics, SIAM Rev. 37 (1995), 53-81.

[7] C. O. Horgan and K. L. Miller, Anti-plane shear deformation for homogeneous and inhomogeneous anisotropic linearly elastic solids, J. Appl. Mech. 61 (1994), 23-29.

[8] A. Matei, V. V. Motreanu and M. Sofonea, A quasistatic antiplane contact problem with slip dependent friction, Adv. Nonlinear Var. Inequal. 4 (2001), 1-21.

[9] D. Motreanu and M. Sofonea, Evolutionary variational inequalities arising in quasistatic frictional contact problems for elastic materials, Abstr. Appl. Anal. 4 (1999), $255-279$.

[10] M. Shillor and M. Sofonea, A quasistatic viscoelastic contact problem with friction, Int. J. Engrg. Sci. 38 (2000), 1517-1533.

[11] M. Sofonea and A. Matei, Elastic antiplane contact problem with adhesion, J. Appl. Math. Phys. (ZAMP) 53 (2002), 962-972.

[12] M. Sofonea and M. Shillor, Variational analysis of a quasistatic viscoplastic contact problem with friction, Commun. Appl. Anal. 5 (2001), 135-151.

Laboratoire de Théorie des Systèmes

Université de Perpignan

52 Avenue de Villeneuve

66860 Perpignan, France

E-mail: sofonea@univ-perp.fr
Laboratoire d'Arithmétique, de Calcul formel et d'Optimisation Université de Limoges 123 Avenue A. Thomas 87060 Limoges, France

E-mail: mohamed.a.mansour@math.net 